\title{
Transcranial direct current stimulation (tDCS): its effect on improving dysphagia in stroke patients
}

\author{
Salah Abd Elmonem Sawan ${ }^{1}$, Abdelazim M. Reda ${ }^{2}$, Ahmed Hosny Kamel ${ }^{3}$ and Mennat Allah Mohamed Ali ${ }^{1}$
}

\begin{abstract}
Background: Transcranial direct current stimulation (tDCS) may have a potential for improving post-stroke dysphagia.

Objective: The purpose of this study was to examine the effect of tDCS on improving dysphagia in stroke patients. Patients and methods: Forty stroke patients were divided randomly into two equal groups (the study (group A) and control groups (group B). Group A received a physical therapy program and active (tDCS), and group B received the same physical therapy program and sham (tDCS). The Dysphagia Outcome and Severity Scale (DOSS) and videofluoroscopy were performed in all patients before and after 2 weeks of the treatment program.

Results: Before treatment, there were no significant differences between the two groups for DOSS score or digital fluoroscopic findings. After treatment, there were significant differences between the study and control group for DOSS score and digital fluoroscopic findings.
\end{abstract}

Conclusion: Anodal tDCS is effective in improving dysphagia in stroke patients.

Keywords: Stroke, Dysphagia, Transcranial direct current stimulation

\section{Introduction}

Dysphagia is a common medical complication that can occur following ischemic stroke and affects $13-94 \%$ of acute stroke patients. Its incidence is related to stroke location and lesion volume [1].

Mortality and morbidity are high in patients with post-stroke dysphagia (PSD) due to aspiration pneumonia and malnutrition. It may improve spontaneously; however, a long-term disability develops in approximately $11.5 \%$ of patients [2].

The pharyngeal phase of swallowing has been shown to be affected in right hemisphere stroke, with impairment the initiation and duration of swallowing and increased rate of aspiration [3]. In contrast, strokes of the left hemisphere result in impairment of pharyngeal

\footnotetext{
*Correspondence: abdelazimneuro@gmail.com

${ }^{2}$ Neurology Department, Faculty of Medicine, Cairo University, Cairo, Egypt Full list of author information is available at the end of the article
}

transit and longer oral transit [4]. Several strategies aimed at modifying corticomotor excitability have emerged which include repetitive transcranial magnetic stimulation (rTMS) and transcranial direct current stimulation (tDCS) [5].

TDCS have some advantages over rTMS, which include long-lasting modulatory effects of cortical function, ease of application, and low cost. In tDCS, a weak direct current is used to stimulate the cerebral cortex [6]. The "long-lasting" effect of tDCS is thought to be related to neuroplastic changes in the brain, its impact on visual perception and cognitive functions, including motor-learning, working memory, episodic, and semantic memory [7].

TDCS has shown to improve the effectiveness of swallowing therapy in patients with dysphagia after stroke. Many studies have found that repeated stimulation of the unaffected swallowing cortex with anodal tDCS in
Springer Open (c) The Author(s). 2020 Open Access This article is licensed under a Creative Commons Attribution 4.0 International License, which permits use, sharing, adaptation, distribution and reproduction in any medium or format, as long as you give appropriate credit to the original author(s) and the source, provide a link to the Creative Commons licence, and indicate if changes were made. The images or other third party material in this article are included in the article's Creative Commons licence, unless indicated otherwise in a credit line to the material. If material is not included in the article's Creative Commons licence and your intended use is not permitted by statutory regulation or exceeds the permitted use, you will need to obtain permission directly from the copyright holder. To view a copy of this licence, visit http://creativecommons.org/licenses/by/4.0/. 
combination with timed effortful swallowing is associated with significant improvement in swallowing compared with sham $\mathrm{tDCS}$ in patients with acute-subacute unilateral hemispheric stroke. This was the case even after adjusting for the effects of other variables such as severity of dysphagia, age of patients, and time of stimulation [8].

The mechanism of tDCS in PSD is depolarization or hyperpolarization of the neural tissue, which induces significant changes in the resting membrane potential and thus modulate synaptic plasticity in ischemic stroke [9].

The purpose of this study was to determine the effectiveness tDCS on improving dysphagia following stroke.

\section{Methods}

This randomized controlled single blinded study was conducted in 40 patients with cerebrovascular ischemic stroke. Patients were selected from (blinded for peer review). The patients' ages ranged from 45 to 60 years. Patients were divided randomly into two equal groups (groups A and B); each comprising 20 patients. Each group was divided into two equal subgroups: group A1 consisted of 10 patients with unilateral hemispheric stroke and group A2 consisted of 10 patients with bilateral hemispheric stroke. Group B1 consisted of $10 \mathrm{pa}-$ tients with unilateral hemispheric stroke and group B2 consisted of 10 patients with bilateral hemispheric stroke.

Patients with acute or subacute ischemic stroke were included in the study. The location of stroke was in the territory of the carotid system and was diagnosed clinically and confirmed by computed tomography and or magnetic resonance imaging of the brain (within the first month). The severity of dysphagia was ranged from mild to severe. Patients were vitally stable, oriented, and were able to follow commands.

Patients were excluded if they had severe impairment of swallowing before the stroke, difficulty communication, impaired cognition, a neuro-degenerative disorder, or major psychiatric illness such as depression, unstable health issues such as severe cardiac disease or renal failure, intracranial devices, and/or metal excluding tDCS application, chronic drug use that could affect brain activity such as anti-epileptics or antipsychotics, alcohol abuse, epilepsy, pregnancy, or a pacemaker or other implanted electrically sensitive device.

\section{Assessment protocol}

Details of each patient were recorded, which included age, gender, time from stroke onset to stimulation and the National Institutes of Health Stroke Scale (NIHSS) as a measure of stroke severity before stimulation.

Swallowing was assessed using different food consistencies (teaspoon, cup sip and straw sip of thin and thick liquids, such as honey and liquidized solids). The Dysphagia Outcome and Severity Scale (DOSS) is a simple and easy-to-apply, 7-point scale to rate the severity of dysphagia, and was obtained before the first session (DOSS-pre) and after the last session (DOSS-post). Videofluoroscopy (VFS), Also known as a modified barium swallow, is the gold standard for swallowing assessments. Oral administration of radio opaque barium is captured in the lateral view with moving images. Occasionally, anterior posterior views may be obtained. The machine used was and Omni Diagnostic Eleva (Philips Medical System Netherlands), which is a device type used for diagnostic X-ray.

\section{Treatment protocol}

Group A1 received anodal tDCS on pharyngeal motor cortex, and a selected physiotherapy program to improve swallowing. For anodal stimulation of the pharyngeal motor cortex, the anode electrode was placed over the healthy hemisphere at mid-distance between C3 and T3 on the left or $\mathrm{C} 4$ and $\mathrm{T} 4$ on the right according to international 10/20 electroencephalogram (EEG) electrode system. A reference electrode was placed over the contralateral supraorbital region. This montage allows a maximal current will be generated over the inferior sensorimotor cortex and the neighboring premotor brain regions that are critical for the reorganization of the swallowing motor cortex in PSD. A constant current of $2 \mathrm{~mA}$ intensity was applied for $30 \mathrm{~min}$. Stimulation was applied for five consecutive sessions for 2 weeks. Group A2 received selected physiotherapy program and active tDCS. Five consecutive sessions were applied on the dominant hemisphere and then on the non-dominant hemisphere. A constant current of $2 \mathrm{~mA}$ intensity was applied for $30 \mathrm{~min}$.

Group B (Control) received the selected physiotherapy program to improve dysphagia and sham tDCS. Stimulation was applied for five consecutive sessions for 2 weeks. The tDCS machine used was an exclusive neuromodulation technology developed by Soterix medical Inc. (New York, USA). The Soterix device has the option of delivering a sham session which causes the patient to feel a similar tingling sensation to active tDCS. This minimizes the placebo effect of the sham condition.

\section{Statistical analysis}

The collected data were analyzed using the statistical package for social studies (SPSS) version 21 for Windows. Data were summarized using mean and standard deviation for quantitative variables if normally distributed, or median if not normally distributed. A percentage was used for qualitative variables. Comparison of treatment effect between different groups was tested using Wilcoxon signed-rank test for quantitative data 
and McNemer for qualitative binomial distribution data. If no variation in esophageal spasm was found before treatment, the effect of treatment was evaluated by fitting the data to a binomial distribution ( 0.05 equal probabilities for response and no response). Correlations had done using Spearman's correlation coefficient. Linear regression analysis was done to detect the predictors of improvement of swallowing. An alpha of 0.05 was used as a level of statistical significance.

\section{Results}

Group A ranged in age from 45 to 60 years with a mean age of $53.30 \pm 5.038$ years. Their NIHSS score ranged from 5 to 20 with a median of 10.50 , and their time to stimulation (day) ranged from 2 to 10 days with a median of 4.50. Group B with ranged from 45 to 60 years with a mean age of $50.30 \pm 5.222$ years. Their NIHSS score ranged from 5 to 20 with a median of 11, their time to stimulation (day) ranged from 2 to 25 days with a median of 7.5. No statistically significant differences between the two groups were found for any of the above parameters $(P>0.05)$ (Table 1$)$.

Results of DOSS score and different components of digital fluoroscopic evaluation pre-treatment and posttreatment in $\mathrm{GA}$

There were significant differences between pre- and post-treatment for all variables $(P<0.05)$ (Table 2$)$.

Results of DOSS score and different components of digital fluoroscopic evaluation pre-treatment and posttreatment in group B

There were no significant differences between preand post-treatment for any of the variables $(P>0.05)$ (Table 3).

Results of DOSS score and different components of digital fluoroscopic evaluation pre-treatment in groups $\mathrm{A}$ and $B$

There were no significant differences between the study and control group for any of the variables before starting treatment $(P>0.05)$ (Table 4$)$.
Results of DOSS score and different components of digital fluoroscopic evaluation post-treatment in groups $A$ and $B$

There were significant differences between the study and control group for all the variables of the DOSS score and different components of the digital fluoroscopic evaluation $(P<0.05)$ (Table 5).

\section{Results of DOSS score and different components of} digital fluoroscopic evaluation pre-treatment for groups A1 and A2

There were no statistically significant differences between the subgroups for any of the variables of the DOSS score or different components of the digital fluoroscopic evaluation $(P>0.05)$. There were also no statistically significant differences between the subgroups for any of the variables $(P>0.05)$ except aspiration, which was improved in the unilateral stroke (group A1; $P<0.05$ ) (Table 6).

\section{Results of DOSS score and different components of} digital fluoroscopic evaluation post-treatment in all patients with unilateral stroke (groups A1 and B1)

There were significant differences between the study and control group for all variables of the DOSS score and different components of digital fluoroscopic evaluation $(P<0.05)$. There were also significant differences between the study and control group in all patients with bilateral stroke (groups A2 and B2) for all variables $(P<$ 0.05 ). The results demonstrated an improvement in patients with unilateral and bilateral stroke.

There was a statistically significant correlation between NIHSS scores, time of stimulation in relation to DOSS score and different components of digital fluoroscopic evaluation $(P<0.05)$. Patients with lower NIHSS score had greater improvement. Furthermore, patients with earlier time of stimulation had greater improvement of all parameters.

Linear regression analysis was done to detect the predictors of improvement of swallowing that was assessed by DOSS score (dependent factor), the NIHSS, time of stimulation were used as an independent predictors. We found that both NIHSS and time of stimulation were

Table 1 Comparison between groups A and B for age, NIHSS score, and time of stimulation

\begin{tabular}{llll}
\hline Demographic data & Group (A) & Group (B) & $P$ value \\
\hline & Mean \pm SD & Mean \pm SD & \\
Age & $53.30 \pm 5.03$ & $50.30 \pm 5.222$ & Median, range \\
NIHSS score & Median, range & & 0.167 \\
& $10.50(5-20)$ & $11(5-20)$ & 0.1 \\
Time to stimulation & $4.50(2-10)$ & $7.50(2-25)$ & 0.186 \\
\hline
\end{tabular}

$P$ probability, *significant $(P<0.05)$

$S D$ standard deviation, *significant $(P<0.05)$

Tests used: Student's $t$ test, Mann-Whitney test 
Table 2 Comparison of the DOSS score and different components of the digital fluoroscopic evaluation between pre- and posttreatment in group A

\begin{tabular}{llll}
\hline Variables & Pre & Post & M value \\
& Median(range) & $6(1-7)$ & $0.001^{*}$ \\
\hline DOSS score & $1(1-3)$ & $20.50(7-50)$ & $0.001^{*}$ \\
Oral transportation time & $44.50(24-55)$ & Frequency (percent) & $17(85 \%)$ \\
& Frequency (percent) & $17(85 \%)$ & $<0.001^{*}$ \\
Positive laryngeal elevation & $4(20 \%)$ & $3(15 \%)$ & $<0.001^{*}$ \\
Positive hyoid elevation & $4(20 \%)$ & $6(30 \%)$ & $0.003^{*}$ \\
Esophageal sphincter spasm & $20(100 \%)$ & & \\
Positive aspiration & $20(100 \%)$ & &
\end{tabular}

DOSS Dysphagia Outcome and Severity Scale

$P$ probability, ${ }^{*}$ significant $(P<0.05)$

Tests used: McNemar test, Wilcoxon signed-rank test, Pearson's chi-squared test

significant predictors for swallowing improvement (Beta $=-0.523, P=0.01),($ Beta $=-0.496, P=0.03)$, respectively.

\section{Discussion}

There are some studies that report favorable effects of rehabilitative interventions and functional recovery following stroke. These include increased perfusion to different cortical areas, reorganization of neuronal plasticity, and recovery of diaschisis. Some functional neuroimaging studies have suggested that reactivation of undamaged portions of the ipsilesional motor cortex is associated with favorable outcomes following stroke. These studies reported that anodal tDCS to both hemispheres may be useful in improving dysphagia following stroke [10].

Jefferson and colleagues [11] stated that anodal tDCS modifies pharyngeal motor cortex excitability, which may affect the swallowing center in the brainstem. Our study showed that the combination of intensive swallowing therapy and anodal tDCS improved swallowing more than swallowing therapy alone.
TDCS has been shown to be a safe intervention with minimal side effects that are limited to tingling, itching, and local erythema [12].

Therapies that combine swallowing exercises with tDCS consider the important mechanism of action to be networking motor outputs with brain stimulation by linking sensory inputs with stimulation. In stroke patients, sensory input from the pharynx has been found to increase the excitability of the swallowing motor cortex of the healthy hemisphere [13].

We showed that there was a significant difference in the median values of the DOSS score between groups A and $B$ at the end of the treatment program. There was also a highly significant difference in the median values of the VFS scores between groups A and the B at the end of the treatment program. Results showed that there was improvement in both unilateral and bilateral lesion patients in group A.

The results of this study is in agreement with the study of Kumar and colleagues [8], who showed that swallowing maneuvers in combination with anodal tDCS improved swallowing in patients with PSD. The study

Table 3 Comparison of the DOSS score and different components of the digital fluoroscopic evaluation between pre- and posttreatment in group B

\begin{tabular}{llll}
\hline Variables & Pre & Post & Median (range) \\
& Median (range) & $2(1-5)$ & 0.773 \\
\hline DOSS score & $2(1-3)$ & $40.50(7-56)$ & 0.116 \\
Oral transportation time & $36(7-55)$ & Frequency (percent) \\
& Frequency (percent) & $5(25 \%)$ & 1 \\
Positive laryngeal elevation & $5(25 \%)$ & $5(25 \%)$ & 1 \\
Positive hyoid elevation & $5(25 \%)$ & $17(85 \%)$ & 0.453 \\
Esophageal sphincter spasm & $14(70 \%)$ & $17(85 \%)$ & 1 \\
Positive aspiration & $16(80 \%)$ & & \\
\hline
\end{tabular}

DOSS Dysphagia Outcome and Severity Scale

$P$ probability, *significant $(P<0.05)$

Tests used: Wilcoxon signed-rank test, Pearson's chi-squared test 
Table 4 Comparison of the DOSS score and different components of the digital fluoroscopic evaluation pre-treatment between both groups A and B

\begin{tabular}{llll}
\hline Variables & $\begin{array}{l}\text { Study group } \\
\text { Median (range) }\end{array}$ & $\begin{array}{l}\text { Control group } \\
\text { Median (range) }\end{array}$ & $\begin{array}{l}\boldsymbol{P} \\
\text { value }\end{array}$ \\
\hline DOSS score & $1(1-3)$ & $2(1-3)$ & $40(15-55)$ \\
Oral transportation time & $36(7-55)$ & Frequency (percent) & 0.09 \\
& Frequency (percent) & $5(25 \%)$ & $5(25 \%)$ \\
Positive laryngeal elevation & $4(20 \%)$ & $14(70 \%)$ & 1 \\
Positive hyoid elevation & $4(20 \%)$ & $16(80 \%)$ & 0.11 \\
Esophageal sphincter spasm & $20(1000 \%)$ & & 0.11 \\
Positive aspiration & $20(100 \%)$ &
\end{tabular}

DOSS Dysphagia Outcome and Severity Scale

$P$ probability, *significant $(P<0.05)$

Tests used: Mann-Whitney test, Pearson's chi-squared test

included 14 patients with unilateral stroke who underwent $\mathrm{tDCS}$ or sham stimulation of the swallowing motor cortex in the unaffected hemisphere for 5 days in combination with swallowing exercises. Dysphagia severity was assessed using the DOSS. Results showed that patients who received anodal tDCS improved by 2.60 points compared with patients who received sham tDCS who improved by1.25 points $(P=.019)$.

Another study using tDCS undertaken by yang and colleagues recruited 16 patients with dysphagia following stroke, who received either anodal or sham tDCS over the pharyngeal motor cortex of the affected hemisphere in combination with 30 min of conventional swallowing exercises, daily for 10 days. Dysphagia severity was assessed using functional dysphagia scale (FDS); scores were measured at baseline, as well as immediately, and 3 months after the intervention. They found that immediately after the intervention, FDS scores had improved to the same degree in both groups. However, after 3 months, the group that had received anodal tDCS showed great improvement in FDS score compared with the sham group. They concluded that anodal tDCS of the affected pharyngeal motor cortex can improve dysphagia outcome in patients with PSD [9].

Also, Shigematsu and colleagues [14] investigated whether tDCS of the pharyngeal motor cortex combined with an intensive swallowing program can improve dysphagia. They enrolled 20 patients who had dysphagia for more than 1 month after stroke. Patients were randomly assigned to receive 10-20-min sessions of either tDCS or a sham procedure to the ipsilesional pharyngeal motor cortex, in combination with a conventional swallowing therapy. The swallowing function was evaluated with DOSS before, immediately after, and 1 month after the last session. The results showed that anodal tDCS resulted in an improvement of 1.4 points in DOSS $(P=$ .006) immediately after the last session and 2.8 points $(P$ $=.004) 1$ month after the last session. The sham tDCS group improved 0.5 points $(P=.059)$ after the last session and 1.2 points $(P=.026) 1$ month after the final session. The improvements in the anodal tDCS group were significantly higher than those in the sham tDCS group $(P=.029$ after the last session, and $P=.007,1$ month after the last session). They concluded that

Table 5 Comparison of the DOSS score and different components of the digital fluoroscopic evaluation post-treatment between groups A and B

\begin{tabular}{llll}
\hline Variables & $\begin{array}{l}\text { Study group } \\
\text { Median (range) }\end{array}$ & $\begin{array}{l}\text { Control group } \\
\text { Median (range) }\end{array}$ & $\begin{array}{c}\boldsymbol{P} \text { value } \\
\text { DOSS score }\end{array}$ \\
Oral transportation time & $6(1-7)$ & $2(1-5)$ & $0.001^{*}$ \\
& $20.50(7-50)$ & $40.50(7-56)$ & $0.004^{*}$ \\
Positive laryngeal elevation & Frequency (percent) & Frequency (percent) & $<(25 \%)$ \\
Positive hyoid elevation & $17(85 \%)$ & $5(25 \%)$ & $<0.001^{*}$ \\
Esophageal sphincter spasm & $17(85 \%)$ & $17(85 \%)$ & $<0.001^{*}$ \\
Positive aspiration & $3(15 \%)$ & $17(85 \%)$ & $0.001^{*}$ \\
\hline
\end{tabular}

DOSS Dysphagia Outcome and Severity Scale

$P$ probability, ${ }^{*}$ significant $(P<0.05)$

Tests used: Mann-Whitney test, Pearson's chi-squared test 
Table 6 Comparison the of DOSS score and different components of the digital fluoroscopic evaluation between pre- and posttreatment in groups $\mathrm{A} 1$ and $\mathrm{A} 2$

\begin{tabular}{|c|c|c|c|c|c|c|}
\hline \multirow[t]{3}{*}{ Variables } & \multicolumn{2}{|l|}{ Group A1 } & \multicolumn{2}{|c|}{ Group A2 } & \multicolumn{2}{|c|}{$P$ value } \\
\hline & \multicolumn{2}{|c|}{ Median (range) } & \multicolumn{2}{|c|}{ Median (range) } & \multirow[b]{2}{*}{ Pre } & \multirow[b]{2}{*}{ Post } \\
\hline & Pre & Post & Pre & Post & & \\
\hline DOSS & $2(1-3)$ & $6(2-7)$ & $1(1-2)$ & $5(1-7)$ & 0.55 & 0.625 \\
\hline \multirow[t]{3}{*}{ Oral transplantation time } & $39(25-55)$ & $29(7-50)$ & $41(24-54)$ & $45(20-50)$ & 0.05 & 0.432 \\
\hline & \multicolumn{2}{|c|}{ Frequency (percent) } & \multicolumn{2}{|c|}{ Frequency (percent) } & & \\
\hline & Pre & Post & Pre & Post & Pre & Post \\
\hline Positive laryngeal elevation & $3(30 \%)$ & $9(90 \%)$ & $1(10 \%)$ & $8(80 \%)$ & 0.352 & 0.109 \\
\hline Positive hyoid elevation & $3(30 \%)$ & $9(90 \%)$ & $1(10 \%)$ & $8(80 \%)$ & 0.352 & 0.109 \\
\hline Esophageal sphincter spasm & 10(100\%) & $1(10 \%)$ & $10(100 \%)$ & $2(20 \%)$ & 1 & 0.344 \\
\hline Positive aspiration & 10(100\%) & $1(10 \%)$ & 10(100\%) & $5(50 \%)$ & 1 & $0.02^{*}$ \\
\hline
\end{tabular}

DOSS Dysphagia Outcome and Severity Scale

$P$ probability, *significant $(P<0.05)$

Pre pre-treatment, Post post-treatment

Tests used: Mann-Whitney test, Pearson's chi-squared test

anodal tDCS to the ipsilesional swallowing motor cortex combined with a swallowing program significantly improved swallowing function in patients with PSD.

In a study done of 60 acute dysphagic stroke patients who received either contralateral anodal or sham tCDS stimulation over the swallowing motor cortex over four consecutive days, a greater improvement in swallowing function was found in the anodal stimulation group. Patients were thoroughly assessed before and after the intervention using the validated fiberoptic endoscopic dysphagia severity scale [15].

There were statistically significant correlations between the NIHSS scores, time of stimulation, DOSS scores, and different digital fluoroscopic evaluation scores. This was in accordance with Suntrup Krueger and colleagues [15], who found that the low NIHSS scores were related to greater swallowing improvement and earlier application of tDCS related to greater swallowing improvement during the acute-subacute stage. Furthermore, the study by Kumar and colleagues [7] that included patients within a similar time frame also obtained positive results.

A study done by Li and colleagues [16] showed that both unilateral and bilateral anodal tDCS combined with conventional therapies are helpful in the recovery of swallowing function in patients with PSD, with bilateral anodal tDCS resulting in substantially more improvement.

In the present study, we overcame the spontaneous recovery of dysphagia in stroke patients by comparing between two groups: unilateral stroke patients who may recover from dysphagia spontaneously, and bilateral stroke patients whose dysphagia was severe and unlikely to recover spontaneously.

The strengths of the present study were the success in starting oral intake without a feeding tube in acute stroke patients with severe dysphagia, and the two tDCS protocols of contralateral and bilateral stimulation.

The limitations of the study were the small sample sizes and the lack of prolonged follow-up. Another limitation was variation in the site of stimulation and tDCS parameters between our and other studies. This prevents the development of an accurate conclusion in this area, and we recommend making head-to-head comparison trials to compare stimulation protocols.

\section{Conclusion}

We concluded that anodal tDCS applied to the contralateral or bilateral hemispheres of acute stroke patients effectively improves swallowing function when combined with intensive swallowing therapy. NIHSS and time of stimulation were significantly correlated with improvement of swallowing and could be used as predictors of improvement of dysphagia after tDCS sessions.

\section{Abbreviations}

DOSS: Dysphagia outcome severity scale; EEG: Electroencephalogram; FDS: Functional dysphagia scale; NIHSS: National institute of health and stroke scale; PSD: Post-stroke dysphagia; rTMS: Repetitive transcranial magnetic stimulation; SPSS: Statistical package for social studies; tDCS: Transcranial direct current stimulation; VFS: Videofluroscopy

\section{Acknowledgements}

The authors acknowledge the subjects for their participation and cooperation in this study.

\section{Authors' contributions}

SAS: research idea, data acquisition, data analysis, and interpretation, AMR: data acquisition, data analysis and interpretation, and manuscript writing and reviewing, AHK: performing video fluoroscopy, MMA: data acquisition, data analysis and interpretation. All authors have read and approved the manuscript.

\section{Funding}

This research received no specific grant from any funding agency in the public, commercial, or not-for-profit sectors. 


\section{Availability of data and materials}

The datasets generated and/or analyzed during the current study are not publicly available due to current Cairo University regulations and Egyptian legislation but are available from the corresponding author on reasonable request and after institutional approval.

\section{Ethics approval and consent to participate}

The aim and procedures of the study were explained to every participant and an informed written consent was obtained from all participants before being enrolled in the study. The study was approved by the ethical committee of Department of Physical Therapy for Neuromuscular Disorders and its Surgery, Faculty of Physical Therapy, Cairo University: NO:P.T.REC/013/ $001081(4 / 10 / 2016)$.

\section{Consent for publication}

Not applicable.

\section{Competing interests}

The authors declare that we have no competing interests (financial and nonfinancial). We declare that the research was conducted in absence of any commercial relationships that could be constructed as a potential conflict of interest.

\section{Author details}

'Department of Physical Therapy for Neuromuscular Disorder and its Surgery, Faculty of Physical Therapy, Misr University for Science and Technology, October City, Egypt. ${ }^{2}$ Neurology Department, Faculty of Medicine, Cairo University, Cairo, Egypt. ${ }^{3}$ Department of Radiology, Faculty of Medicine, Cairo University, Cairo, Egypt.

Received: 19 June 2020 Accepted: 13 November 2020

Published online: 26 November 2020

\section{References}

1. Langdon PC, Lee AH, Binns CW. Dysphagia in acute ischaemic stroke: severity, recovery and relationship to stroke subtype. J Clin Neurosci. 2007; 14(7):630-4

2. Cohen DL, Roffe C, Beavan J, Blackett B, Fairfield CA, Hamdy S, et al. Poststroke dysphagia: A review and design considerations for future trials. Int J Stroke. 2016:4:399-411.

3. Robbins J, Levine RL, Maser A, Rosenbek JC, Kempster GB. Swallowing after unilateral stroke of the cerebral cortex. Arch Phys Med Rehabil. 1993;74(12): 1295-300.

4. Miller AJ. The neurobiology of swallowing and dysphagia. Dev Disabil Res Rev. 2008:14(2):77-86.

5. Nitsche MA, Paulus W. Excitability changes induced in the human motor cortex by weak transcranial direct current stimulation. J Physiol. 2000;527(3): 633-9.

6. Pascual-Leone A, Valls-Solé J, Wassermann EM, Hallett M. Responses to rapid-rate transcranial magnetic stimulation of the human motor cortex. Brain. 1994;117(4):847-58.

7. Jacobson L, Koslowsky M, Lavidor M. TDCS polarity effects in motor and cognitive domains: a meta-analytical review. Exp Brain Res. 2012;216(1):1-10.

8. Kumar S, Wagner CW, Frayne C, Zhu L, Selim M, Feng W, et al. Noninvasive brain stimulation may improve stroke-related dysphagia: A pilot study. Stroke. 2011;42(4):1035-40.

9. Yang EJ, Baek SR, Shin J, Lim JY, Jang HJ, Kim YK, et al. Effects of transcranial direct current stimulation (tDCS) on post-stroke dysphagia. Restor Neurol Neurosci. 2012;30(4):303-11.

10. Fregni F, Boggio PS, Lima MC, Ferreira MJ, Wagner T, Rigonatti SP, et al. A sham-controlled, phase II trial of transcranial direct current stimulation for the treatment of central pain in traumatic spinal cord injury. Pain. 2006; 122(1-2):197-209.

11. Jefferson S, Mistry S, Singh S, Rothwell J, Hamdy S. Characterizing the application of transcranial direct current stimulation in human pharyngeal motor cortex. Am J Physiol Gastrointest Liver Physiol. 2009;297(6):G1035-40.

12. Schlaug $G$, Renga $V$, Nair D. Transcranial direct current stimulation in stroke recovery. Arch Neurol. 2008;65(12):1571-6.

13. Khedr EM, Abo-Elfetoh N. Noninvasive brain stimulation for treatment of post-stroke dysphagia. Neuroenterology. 2013;2:1-9.
14. Shigematsu T, Fujishima I, Ohno K. Transcranial direct current stimulation improves swallowing function in stroke patients. Neurorehabil Neural Repair. 2013;27(4):363-9.

15. Suntrup Krueger S, Ringmaier C, Muhle P, Wollbrink A, Kemmling A, Hanning $U$, et al. Randomized trial of transcranial direct current stimulation for poststroke dysphagia. Ann Neurol. 2018;83(2):328-40.

16. Li Y, Feng H, Li J, Wang H, Chen N, Yang J. The effect of transcranial direct current stimulation of pharyngeal motor cortex on swallowing function in patients with chronic dysphagia after stroke. A retrospective cohort study. Med. 2020;99:e19121.

\section{Publisher's Note}

Springer Nature remains neutral with regard to jurisdictional claims in published maps and institutional affiliations.

\section{Submit your manuscript to a SpringerOpen ${ }^{\circ}$ journal and benefit from:}

- Convenient online submission

- Rigorous peer review

- Open access: articles freely available online

High visibility within the field

- Retaining the copyright to your article

Submit your next manuscript at $\boldsymbol{\sim}$ springeropen.com 